\title{
Application of PNN Optimized by MEA in GIS Partial Discharge Recognition
}

\author{
Ya Li ${ }^{1, a,}$, Haoyang Cui ${ }^{1, * b}$, Gaofang $\mathrm{Li}^{1, \mathrm{c}}$, Yongpeng $\mathrm{Xu}^{2, \mathrm{~d}}$ \\ College of Electronics and Information Engineering, ${ }^{1}$ Shanghai University of Electric Power, Shanghai, \\ China. ${ }^{2}$ Shanghai University of Electric Power, Shanghai, China. \\ a email: 1319048074@qq.com, bemail: cuihy@shiep.edu.cn \\ ce-mail: lioneix_1128@sina.com, ${ }^{\mathrm{d}}$ email: 441386758@qq.com \\ *corresponding author
}

Keywords: Mind Evolutionary Algorithm, Gas Insulated Switchgear, Probabilistic Neural Network, Discharge recognition

\begin{abstract}
Aiming at the problem that the probabilistic neural network (PNN) is difficult to determine the smoothing factors in the process of partial discharge recognition in GIS. A model of GIS partial discharge recognition based on Mind evolutionary algorithm (MEA) is proposed to optimize the PNN. The MEA has the strong ability of searching, obtaining the global approximate optimal solution, finding the optimal smoothing factor of PNN, and improving the accuracy of partial discharge classification. In order to verify the validity and practicability of this model, the simulations are carried out using three typical discharge defect samples. Compared with back propagation (BP) neural network and PNN, the results show that the partial discharge recognition accuracy and stability of PNN optimized by MEA are better and with certain research value.
\end{abstract}

\section{Introduction}

Gas Insulated Switchgear (GIS) is widely used in power systems with many advantages. If the GIS occurs failure may lead to switch failure, a long time and widespread blackouts. Also it may affect the entire power system. Insulation defects account for the largest proportion of various factors that cause failure of GIS, the partial discharge patterns of various insulation defects are obviously different ${ }^{[1-2]}$. By recognizing the partial discharge, we can diagnose the type of insulation defects, evaluate the insulation state, prevent and avoid the further deterioration of the insulation defects, and prepare the maintenance plan to ensure the safe and stable operation of GIS ${ }^{[3]}$.The traditional methods of partial discharge recognition are mainly through the experienced experts to observe the discharge spectrum. However, it is inefficient and error-prone. With the development of artificial neural network, back propagation (BP) network with simple structure, strong nonlinear approximation ability and generalization ability is widely used in partial discharge recognition, which improves the speed and accuracy of partial discharge recognition. Compared with BP network, probabilistic neural network (PNN) is mainly used in the fields of pattern recognition and fault diagnosis because of its fast training speed and difficult to fall into local minimum. But the smoothing factors closely related to the classification results are difficult to be determined. The process of empirical calculation is complicated. With further research, the corresponding references ${ }^{[4-5]}$ use the genetic algorithm and the particle swarm optimization algorithm to optimize the smoothing factor of the PNN. However, the sample data are large in the process of GIS partial discharge recognition. Nevertheless, two algorithms mentioned above have low accuracy and susceptible to premature convergence because of the initial population size, which affects the accuracy of partial discharge recognition. Then, this paper adopts the mind evolutionary algorithm (MEA) based on the genetic algorithm with two mutually convergent convergence and alienation operators, which can search the optimal individuals quickly in order to find the optimal smoothing factors and improve the recognition rate of GIS partial discharge. 


\section{The Model of GIS Partial Discharge Recognition Based on PNN Optimized by MEA}

\subsection{The Overview of Probabilistic Neural Network}

PNN mainly used for pattern classification is a forward feedback neural network based on Bayesian strategy. As shown in Fig.1 It is composed of input layer, pattern layer, sum layer and contest layer ${ }^{[6]}$.In the model of PNN, the number of neurons in the input layer are equal to the input vector $m$ in the learning samples. Each neuron is a simple distribution unit that passes the input variables directly to the pattern layer. The number of nodes in the pattern layer are determined by the input samples and the type which is defined as $m \times L$. At the pattern layer, the outputs are weighted summation of inputs. Then, after the operation of an activation function, it is passed to the sum layer ${ }^{[7-8]}$

$$
\theta_{i}=\exp \left(-\sum\left(\left\|x-c_{i}\right\|^{2} / 2 \sigma_{i}^{2}\right)\right)
$$

Where $c_{i}$ is the center of the radial basis function, which represents the switching parameter of the $i$-th component of the characteristic function.

The units of the sum layer are only connected to the corresponding model units. Each unit only estimates the probability of each classification according to the parzen method. The conditional probability is:

$$
P\left(X \mid C_{i}\right)=\frac{1}{\left(2 \prod\right)^{m / 2} \sigma^{m}} \frac{1}{n} \sum_{i=1}^{n} \exp \left[-\frac{\left(X-X_{i}\right)^{T}\left(X-X_{L}\right)}{2 \sigma^{2}}\right]
$$

Where $C_{i}$ is the sample class, $X$ is the recognition sample. $X_{i}$ is pattern samples for category $i . m$ is the vector dimension. $\sigma$ is smoothing parameters. $n$ is the number of pattern samples for category $i$. The prior probability is recorded as $P(X)$.

The number of decision nodes is equal to the number of category matched. According to the estimation of the input vector probability, Bayes classification rule is used to select the classification with the smallest risk. That is, the category with the maximum a posteriori probability and the decision-making method can be expressed by the following formula:

$$
P\left(X \mid C_{i}\right) P\left(C_{i}\right)>P\left(X / C_{j} P\left(C_{j}\right)\right)
$$

The output is $\mathrm{y}(X)=C_{i}$. Compared with other methods, PNN can converge to Bayesian optimal solution without sufficient computation. In the case of training mode samples, it is only need to adjust the smoothing factor and the network converge fast. The value of the smoothing factor determines influence degree between the pattern points, which is related to the change in the probability density distribution function ${ }^{[9-10]}$ In general, the network only requires an empirical reference to set a smoothing factor. The value of $\sigma$ is too small for the training of the sample just to play the role of isolation. In essence, it is the nearest neighbourhood classifier. If the smoothing factor $\sigma$ is too large to distinguish the details completely, it may not be the ideal classification effect for the boundaries of the different categories, and close to the linear classification at this point. Therefore, how to determine the appropriate parameter of $\sigma$ is the key problem of probabilistic neural networks.

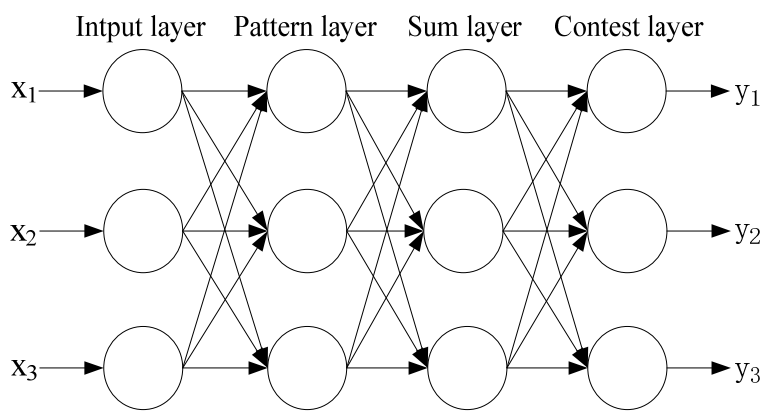

Fig.1 The structure of PNN 


\subsection{Probabilistic Neural Network Optimized by MEA}

The MEA with the ability of global search is used to optimize the PNN, which solves the problem that the classification accuracy of GIS partial discharge is not high because of the improper selection of PNN smoothing factor.

The PNN with three typical defect discharge types is constructed based on the extracted six characteristic parameters. We need to initialize the population and encode the connection weight between the input layer and the competition layer of the PNN by real number coding ${ }^{[11]}$, select the reciprocal of the mean square error of the partial discharge recognition result as the score function, and take the high score as the center of the new population. It can prompt the MEA to iterate ${ }^{[12]}$, through the convergence and dissimilation operation. When the number of iterations reach to the set value, the individual with the highest score is decoded as the best smoothing factor of PNN, which used to retrain the network.

The basic idea about the connection weights optimized by MEA is that firstly a group of individuals is searched in parallel with MEA and form a sequence of evolving populations. The global optimal solution is obtained by some evaluation method and then the neural network is designed by using these good solutions. At last, the optimal solution of the problem is obtained quickly. The concrete steps are as follows ${ }^{[13-14]}$ :

(1) Set the range of smoothing factor, generate the initial population randomly, $M$ is the population size, and set the current algebra to 1 ;

(2) The PNN is constructed according to the smoothing factor obtained by the individual highest score, and calculate the reciprocal of classification error, that is, the fitness function of the highest individual score;

(3) According to the fitness convergence, the dissimilation operations are used to generate new populations representing solutions;

(4) Set a certain number of iterations and search for the highest scores of individuals S sub populations after N-iterations. The initial parameters of the PNN are determined by using the highest individual S-score as the initial solution;

(5) Using the optimized smoothing factor to determine the PNN model, we input the characteristic data extracted from the partial discharge spectrum of GIS as the test data to identify the partial discharge type of GIS;

(6) After training neural network many times, the calculation is stopped according to the specified accuracy to meet the requirements;

In the MEA computation, the real number coding method is adopted. The whole populations are divided into a number of initial sub-groups, in accordance with the principle of survival of the fittest one by one generation to produce better and better approximate solution. In each generation, individuals are selected according to the degree of fitness of the individual, and through the "convergence" and "alienation" operations the species representing the new solution groups are generated. This process will lead to a greater adaptation of the population to the environment. The optimal individual in the last population can be used as the approximate optimal solution of the problem.

\section{The Simulation Experiment of Probabilistic Neural Network Optimized by MEA}

\subsection{Extraction of Feature Parameters}

There are three main types of partial discharge in the interior of GIS: the metal spikes, the discharge of free metal particles, and the discharge of suspended electrodes. Using the statistical parameter method extract parameters such as skewness $S_{k}$, steepness $K_{u}$, correlation coefficient $c c$, discharge factor $Q$, phase asymmetry $\varphi$ and corrected mutual correlation coefficient $m c c$ of the discharge sample. According to the extracted characteristic parameters, the PNN with six inputs and three outputs is constructed. The outputs of the neural network represents the metal tip spikes, the discharge of free metal particles, and the suspended electrode discharge, respectively. The 3-D PD spectrum for three types of GIS defect models are shown in Fig.2 . 
(a)

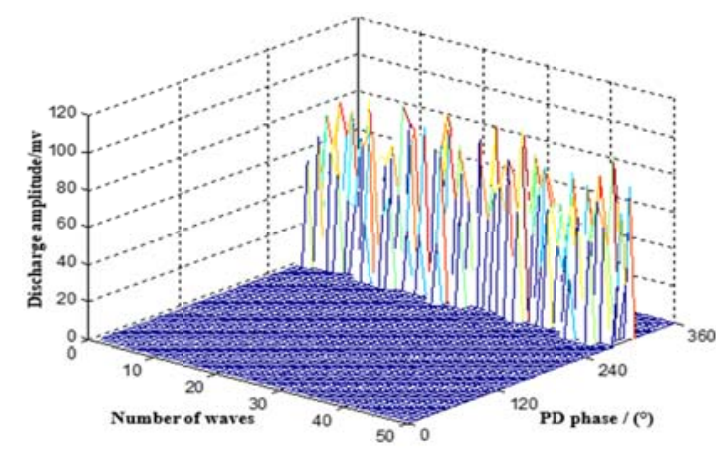

(b)

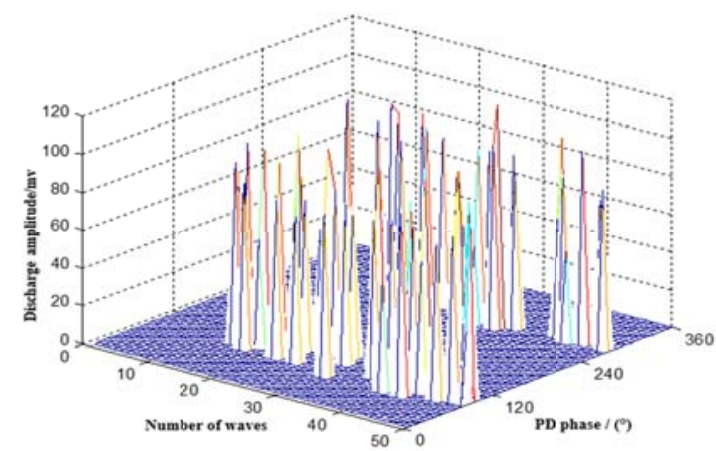

(c)

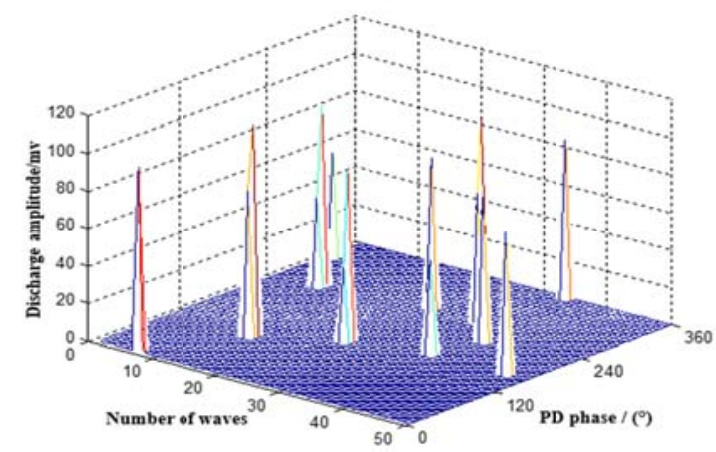

Fig. 2 3-D PD spectrum for three types of GIS defect models

(a) Metal tip discharge; (b) Free metal particles discharge; (c) Suspended electrode discharge

\subsection{Simulation Results of PNN Optimized by Mind Evolutionary Algorithm}

The simulation results are shown in Fig.3 (a)-(c), and the red lines in the figure represent the expected discharge type, and the blue line represent simulation discharge type. If the red line and blue line completely coincide, the discharge recognition is correct. Otherwise, discharge recognition is error. Fig.3(a)-(c) shows that the number of partial discharge error recognition of BP network, PNN, and PNN optimized by MEA model is 4, 3 and 1 respectively. Obviously the accuracy of MEA-PNN is the highest in the three partial discharge recognition models.

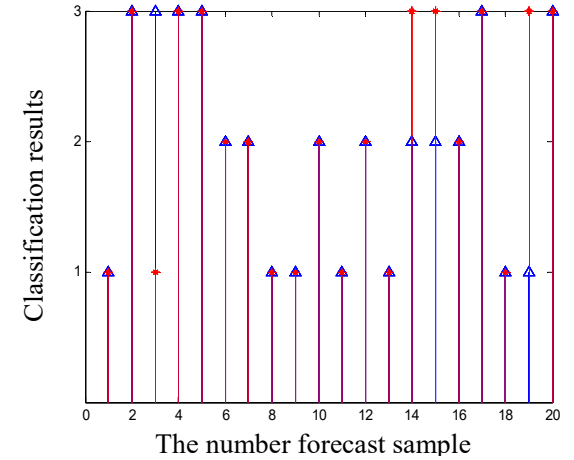

(a) The recognition results of BP network

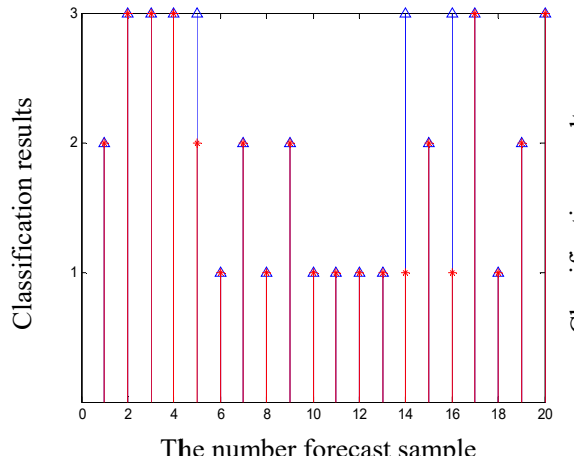

(b) The recognition results of PNN

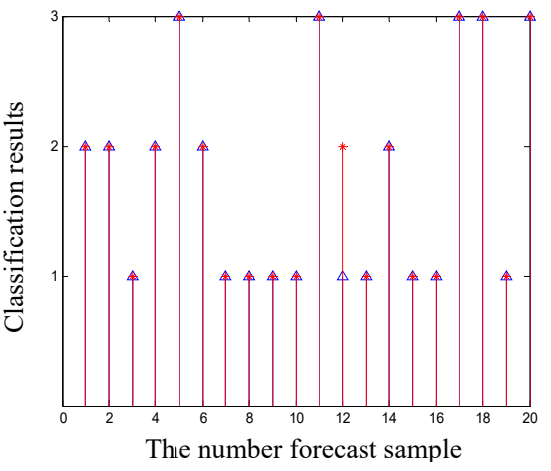

(c) The recognition results of MEA-PNN

Fig.3 Partial discharge recognition results of three network

The results of the above three models for GIS partial discharge type are shown in Table 1, and 
the average recognition accuracy is $80 \%, 85 \%$ and $95 \%$, respectively. From the simulation results, the accuracy of the discharge recognition based on PNN model is much higher than that of the former two, and the results of the partial discharge recognition are more reliable. The reason of the difference in the accuracy of the three models is as follows: the BP network needs to make the network structure fine and complicated in order to make full use of the training data, however, there is a large randomness in the sample training data and the number of samples is small. In order to make full use of the training data, the structure of BP network should be refined to achieve the partial discharge recognition of the training data correctly. However, the complex network structure may lead to over-fitting, which leads to the low accuracy of partial discharge recognition. Compared with the BP network, the PNN is simple in structure and has strong fault tolerance. The partial discharge recognition accuracy is high. MEA-PNN model can find the best smoothing factor from the global, which solves the incorrect selection problem of PNN on the recognition rate of GIS partial discharge, which greatly improves the accuracy of discharge recognition.

Table1 Discharge recognition results of four partial discharge models

\begin{tabular}{|c|c|c|c|c|}
\hline $\begin{array}{l}\text { Partial discharge } \\
\text { recognition model }\end{array}$ & Discharge type & $\begin{array}{l}\text { The number of } \\
\text { misrecognition }\end{array}$ & Accuracy & $\begin{array}{l}\text { Average } \\
\text { accuracy }\end{array}$ \\
\hline \multirow{3}{*}{ BP Network } & Metal tip discharge & 1 & 85.714 & \multirow{3}{*}{80} \\
\hline & $\begin{array}{c}\text { Free metal particles } \\
\text { discharge }\end{array}$ & 2 & 60.000 & \\
\hline & $\begin{array}{c}\text { Suspended electrode } \\
\text { discharge }\end{array}$ & 1 & 87.500 & \\
\hline \multirow{3}{*}{ PNN } & Metal tip discharge & 2 & 77.778 & \multirow{3}{*}{85} \\
\hline & $\begin{array}{c}\text { Free metal particles } \\
\text { discharge }\end{array}$ & 1 & 83.333 & \\
\hline & $\begin{array}{l}\text { Suspended electrode } \\
\text { discharge }\end{array}$ & 0 & 100.000 & \\
\hline \multirow{3}{*}{$\begin{array}{l}\text { PNN optimized by } \\
\text { MEA }\end{array}$} & Metal tip discharge & 0 & 100.000 & \multirow{3}{*}{95} \\
\hline & $\begin{array}{c}\text { Free metal particles } \\
\text { discharge }\end{array}$ & 0 & 100.000 & \\
\hline & $\begin{array}{c}\text { Suspended electrode } \\
\text { discharge }\end{array}$ & 1 & 88.889 & \\
\hline
\end{tabular}

\section{Conclusion}

BP network is easy to fall into local minimum, which leads to unstable discharge recognition and low accuracy. The PNN based on Bayesian decision rules has the advantages of simple structure and difficult to fall into the limit value. And the recognition accuracy of the partial discharge recognition is better than that of the $\mathrm{BP}$ network. Although the convergence speed and recognition accuracy are better than BP network, there is a problem that the smoothing factor is difficult to be determined. When the PNN model is optimized by the mind evolutionary algorithm, the optimal initial weights are obtained from the global search by the "convergence" and "alienation" operators, which are unique to the mind evolutionary algorithm. In this method, it is not only improve the convergence speed of the network, but also improve the accuracy of GIS special high-frequency partial discharge classification. The experiments show that the model of PNN model optimized by MEA designed for partial discharge of GIS is more successful and can be used to recognize the partial discharge type. 


\section{Acknowledgements}

This work was supported by the NSFC (61107081, 11647023), Shanghai Local Colleges and Universities capacity Building Program (15110500900), Shanghai Natural Science Foundation, China (17ZR1411500), and Intelligent Power Grid Center Knowledge Service Platform Achievement Cultivation Project (A-0010-16-029-001).

\section{References}

[1] Li Lixue, Teng Letian, Huang Chengjun, Zeng Yi, Jiang Xiuchen. Envelope Analysis and Defects recognition of Partial Discharge UHF Signals in GIS [J]. High Voltage Technology, 2009, (02): 260-265.

[2] Gao W, Ding D, Liu W, et al. Investigation of the Evaluation of the PD Severity and Verification of the Sensitivity of Partial-Discharge Detection Using the UHF Method in GIS [J]. IEEE Transactions on Power Delivery, 2014, 29(29): 38-47.

[3] Dai D, Wang X, Long J, et al. Feature extraction of GIS partial discharge signal based on Stransform and singular value decomposition [J]. Iet Science Measurement \& Technology, 2017, 11(2):186-193.

[4] Peng Pai, Zhou Yusheng, Gao Yunlong, et al. Ground Fault Line Selection Method in Distribution Network Using GA Optimal LVQ Network [J]. Proceedings of the CSU-EPSA, 2015, 27(10): 64-69.

[5] Cheng Y F, Shao W, Zhang S J, et al. An Improved Multi-Objective Genetic Algorithm for Large Planar Array Thinning [J]. IEEE Transactions on Magnetics, 2016, 52(3): 1-4.

[6] Lian Y, Li G, Wu F, et al. Fault-diagnosis method for INS/GPS integrated navigation system based on PNN and genetic algorithm [J]. Chinese Journal of Scientific Instrument, 2012, 33(1):120-126.

[7] Yang Banghua, Yan Guozheng.Improving the Recognition Rate of EEG in BCI Based on Genetic Algorithm and Probabilistic Neural Network [J]. Journal of Shanghai Jiaotong University, 2005, 39 (10): 1689-1693.

[8] Li Qingquan, Wang Wei, Wang Xiaolong.Fault Diagnosis of Oil immersed Power Transformer by DGA- NN [J]. High Voltage Engineering, 2007, 33 (8): 48-51.

[9] Lin C T, Prasad M, Saxena A. An Improved Polynomial Neural Network Classifier Using RealCoded Genetic Algorithm [J]. IEEE Transactions on Systems Man \& Cybernetics Systems, 2015, 45(11):1389-1401.

[10]Wang Zhongmin, Zhou Peng, Li Gang. Fault Diagnosis of Roller Bearing Based on an Improved Probabilistic Neural Network [J]. Mechanical Soience and Technology for Aerospace Engineering, 2013, 32 (5): 111-114.

[11]T.Mangayarkarasi, D. Najumnissa Jamal. PNN-based analysis system to classify renal pathologies in Kidney Ultrasound Images[C]// Computing and Communications Technologies (ICCCT), 2017 2nd International Conference on. IEEE, 2017.

[12]Gao J L, Gao Q. Research and Application of Rough Set-BP Neural Network Based on MEA [J]. Science Technology \& Engineering, 2011, 11(23): 5564-5568.

[13]Li Xiuguang. Fault Diagnosis for Power Transformer Based on Neural Network Optimized by Mind Evolution Algorithm [D]. Taiyuan University of Technology, 2010.

[14]Wang Xiaochuan. MATLAB Neural Network Analysis of 43 Cases [M]. Beijing University of Aeronautics and Astronautics Press, 2013: 256-261. 